\title{
ASSOCIATION OF CANADIAN UNIVERSITY DEPARTMENTS OF ANESTHESIA
} POSTGRADUATE FELLOWSHIPS IN ANESTHESIA

\begin{tabular}{|c|c|c|c|}
\hline University & Position & Next Available & Contact \\
\hline $\begin{array}{l}\text { Dalhousie University } \\
\text { Halifax, Nova Scotia }\end{array}$ & $\begin{array}{l}\text { 2-Anesthesia Research } \\
\text { 1-Chronic Pain Management } \\
\text { 1-Acute Pain Management } \\
\text { l-Obstetric } \\
\text { l-Pediatric }\end{array}$ & $2000 / 2001$ & $\begin{array}{l}\text { Dr. T.J. Coonan, } \\
\text { Department of Anesthesia, QEII Health Sciences Centre, Victoria General Site } \\
1278 \text { Tower Road, Halifax, Nova Scotia B3H 2Y9, Phone: }(902) 473-2331\end{array}$ \\
\hline $\begin{array}{l}\text { McGill University } \\
\text { Montréal, Québec }\end{array}$ & $\begin{array}{l}\text { 1-Cardiovascular } \\
\text { 2-Obstetric } \\
\text { 1-Pain Management } \\
\text { 2-Pediatric } \\
\text { 1-Neuroanesthesia } \\
\text { 1-Clinical/Research } \\
\text { 1-Thoracic }\end{array}$ & $2000 / 2001$ & $\begin{array}{l}\text { Dr. Francesco Carli, Chairman, } \\
\text { Department of Anesthesia, McGill University, } \\
\text { Royal University Hospital, } 687 \text { Pine Avenue West, Room F9.16, } \\
\text { Montreal, Quebec H3A lAl, Phone: (514) 842-1231, Local } 5342\end{array}$ \\
\hline \multirow[t]{5}{*}{$\begin{array}{l}\text { University Health } \\
\text { Network (Toronto } \\
\text { General, Western } \\
\text { hospitals, Princess } \\
\text { Margaret Hospital) } \\
\text { and Mt. Sinai Hospital }\end{array}$} & $\begin{array}{l}\text { 2-Thoracic (Clinical/Research) } \\
\text { 8-Cardiac \& Intensive Care } \\
\text { (ICU/Clinical/ Research) } \\
\text { 4-Pain (Acute/Chronic/Clinical } \\
\text { Research) } \\
\text { 3-Regional Anesthesia } \\
\text { (Clinical/Rescarch) } \\
\text { 2-Transplantation } \\
\text { (Heart/Lung/Liver) } \\
\text { 4-Neurosurgical } \\
\text { (Clinical/Research) } \\
\text { 2-Ambulatory Anesthesia } \\
\text { (Clinical/Research) } \\
\text { 4-Clinical Anesthesia } \\
\text { 4-Obstetrical } \\
\text { (Clinical/Research - } \\
\text { Mt. Sinai Hospital) }\end{array}$ & $2000 / 2001$ & $\begin{array}{l}\text { Dr. Alan N. Sandler, Anesthesiologist-in-Chief, Department of Anesthesia, } \\
\text { University Health Network and Mt. Sinai Hospital, } 585 \text { University Avenue, } \\
\text { Toronto, Ontario, Canada.M5G 2C4 } \\
\text { Fax: (416) } 340-3698 \text {, Phone: (416) } 340-3275 \text {, } \\
\text { E-mail: asandler@torhosp.toronto.ca }\end{array}$ \\
\hline & $\begin{array}{l}\text { 1-Obstetrical } \\
\text { (Clinical/Research) }\end{array}$ & $2000 / 2001$ & $\begin{array}{l}\text { Dr. S. Halpern, Director of Obstetrical Anesthesia, Department of Anaesthesia, } \\
\text { Women's College Hospital, } 76 \text { Grenville Street, } \\
\text { Toronto, Ontario, Canada M5S IB2, Phone: (416) 323-6269 }\end{array}$ \\
\hline & $\begin{array}{l}\text { 1-Clinical/Research } \\
\text { 8-Pediatric/Anesthesia } \\
\text { (Clinical/Research/Pain } \\
\text { management) }\end{array}$ & $\begin{array}{l}\text { Jan or July } \\
2000 \text { for } 1 \text { yr } \\
2000 / 2001\end{array}$ & $\begin{array}{l}\text { Dr. Jean Kronberg, Anesthetist-in-Chief, Women's College Hospital, } \\
76 \text { Grenville St., Toronto, Ontario, M5S 1B2, Phone: (416) 323-6008 } \\
\text { Dr. L. Roy, Anesthetist-in-Chief, Department of Anesthesia, Hospital } \\
\text { for Sick Children, 555 University Avenue, Toronto, Ontario, Canada M5G 1X8 } \\
\text { Phone: (416) 813-7445, Fax: (416) 813-7543, E-mail: roy@anes.sickkids.on.ca } \\
\text { Information available at: http://www.sickkids.on.ca/anesthesia }\end{array}$ \\
\hline & $\begin{array}{l}\text { 8-Pediatric Critical Care } \\
\text { (Clinical) } \\
\text { 2-Pediatric Critical Care } \\
\text { (Research) }\end{array}$ & $2000 / 2001$ & $\begin{array}{l}\text { Dr. G.A. Barker, Chief, Department of Critical Care Medicine, } \\
\text { Hospital for Sick Children, } 555 \text { University Avenue, Toronto, } \\
\text { Ontario, Canada M5G 1X8, Phone: (416) 813-6860 }\end{array}$ \\
\hline & $\begin{array}{l}\text { 1-Trauma/Critical Care } \\
\text { (Clinical/Research) } \\
\text { 3-Clinical/Research Anesthesia } \\
\text { 1-Critical Care (Clinical/Rescarch) } \\
\text { 1-Cardiac (Clinical/Research) } \\
\text { 1-Regional Anesthesia } \\
\text { (Clinical/Research) } \\
\text { 6-Pain/Critical Care/Trauma/ } \\
\text { Cardiac (including echocardio- } \\
\text { graphy)/Neuro or customized } \\
\text { program (Clinical Research) }\end{array}$ & $2000 / 2001$ & $\begin{array}{l}\text { Dr. J.H. Devitt, Anesthetist-in-Chief, Department of Anesthesia, Sunnybrook } \\
\text { Health Sciences Centre, 2075 Bayview Avenue, M3-200, Toronto, Ontario } \\
\text { M4N 3M5, Phone: (416) 480-4864, Fax: (416) 480-6039, } \\
\text { E-mail: hugh.devitt@sunnybrook.on.ca } \\
\text { Dr. D.K. Rose, Anesthetist-in-Chief, Department of Anesthesia, } \\
\text { St. Michael's Hospital, 30 Bond Street, Toronto, Ontario, Canada, M5B lW8 } \\
\text { Phone: (416) 864-5071, Fax: (416) 864-6014, E-mail: rosek@smh.roronto.on.ca } \\
\text { More detailed information on these fellowship positions may be obtained from the } \\
\text { Department of Anaesthesia home page on the WWW at } \\
\text { http://www.canmed.utoronto.ca/anaesthesia }\end{array}$ \\
\hline \multirow[t]{3}{*}{$\begin{array}{l}\text { University of Ottawa, } \\
\text { Ottawa, Ontario }\end{array}$} & $\begin{array}{l}\text { 1-Chronic Pain Management } \\
\text { 1-Obstetrical Anesthesia } \\
\text { 1-Regional Anesthesia } \\
\text { 1-Cardiac Anesthesia }\end{array}$ & $\begin{array}{l}\text { January } 2000 \\
\text { January } 2000 \\
\text { July } 2000\end{array}$ & $\begin{array}{l}\text { Dr. Dennis Reid, Head, Department of Anesthesia, Ottawa Hospital - Alta Vista } \\
\text { Site, 501 Smyth Road, Ottawa, Ontario KIH 8L6, Phone: (613) 737-8187 } \\
\text { Dr. J. Robblee, Head, University of Ottawa Heart Institute, } \\
40 \text { Ruskin, Ottawa. Phone: (613) 761-4379 }\end{array}$ \\
\hline & $\begin{array}{l}\text { 1-Obstetric Anesthesia } \\
\text { 1-Chronic Pain } \\
\text { 1-Vascular Anesthesia } \\
\text { 1-Thoracic }\end{array}$ & & $\begin{array}{l}\text { Dr. Larry Byford, Chief, Department of Anesthesia, B30, Ottawa Hospital - Civic } \\
\text { Site, } 1053 \text { Carling Avenue, Ottawa, Ontario KIY 4E9, Phone: (613) 761-4940 }\end{array}$ \\
\hline & 2-Pediatric Anesthesia & January 2000 & $\begin{array}{l}\text { Dr. William Splinter, Chief, Department of Anesthesia, Children's Hospital of } \\
\text { Eastern Ontario, } 401 \text { Smyth Road, Ottawa, Ontario, Phone: (613) 737-2431 }\end{array}$ \\
\hline $\begin{array}{l}\text { University of Calgary } \\
\text { Calgary, Alberta }\end{array}$ & 1-Pediatric & $2000-2001$ & $\begin{array}{l}\text { Dr. G.V. Goresky, Acting Regional Clinical Department Head, Department of } \\
\text { Anesthesia, Foothills Hospiral, The University of Calgary, 1403-29th St. N.W., } \\
\text { Calgary, Alberta T2N } 2 \text { T9 Phone: (403) } 670-1430\end{array}$ \\
\hline
\end{tabular}


ASSOCIATION OF CANADIAN UNIVERSITY DEPARTMENTS OF ANESTHESIA POSTGRADUATE FELLOWSHIPS IN ANESTHESIA

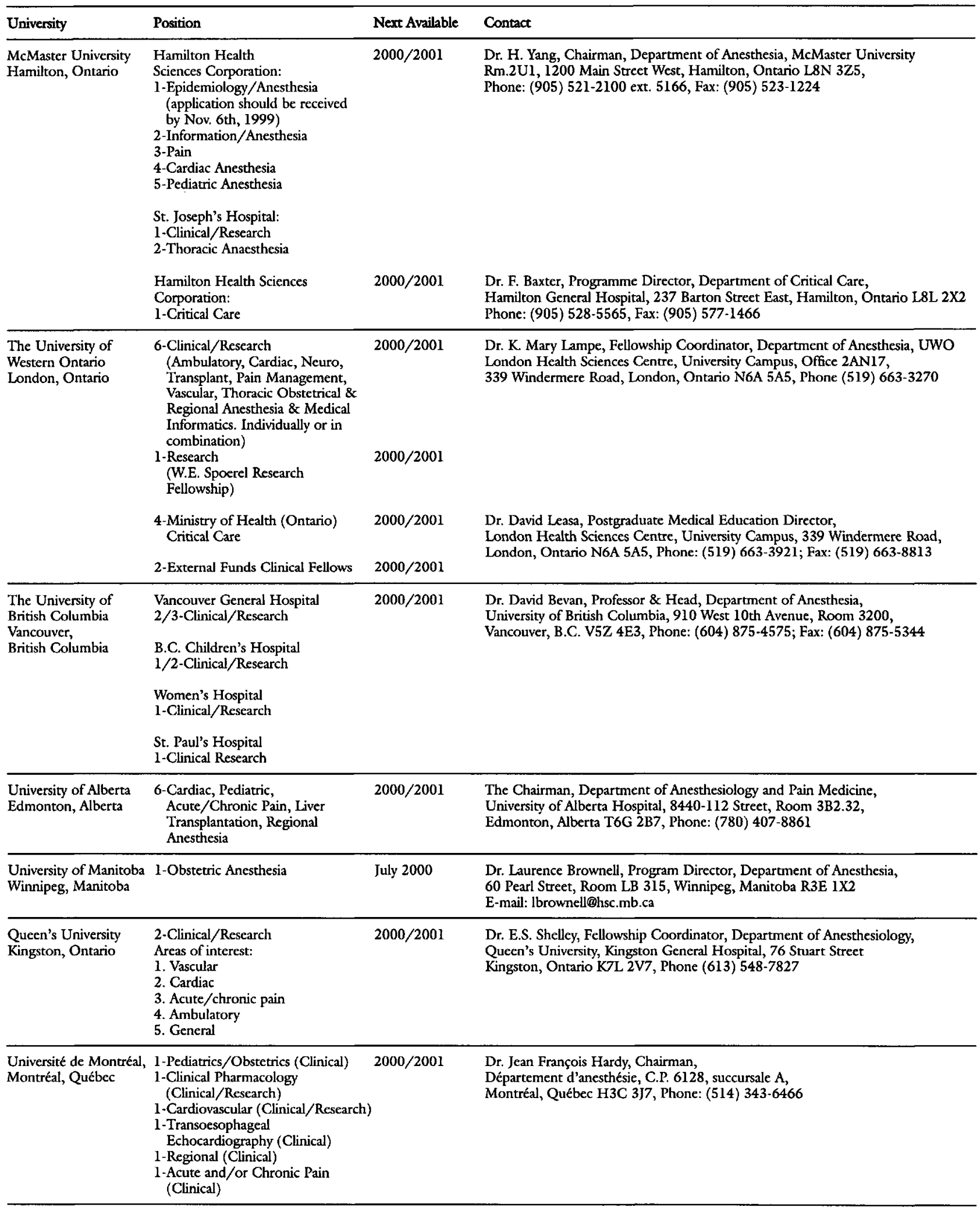

Footnotes 1. Qualifications: FRCPC or eligibility for examinations, except Vancouver General Hospiral (completed minimum 3 years in Resident programme) and University of Toronto Grant Dependent Positions (minimum 2 years in Resident programme).

2. Remuneration, Medical Licencing Requirements, and other conditions: Contact individual departments.

3. Fellowships available for Canadian Cirizens or Landed Immigrants.

4. Two-year programme; possible one-year overlap with base programme of anaesthesia. Required: FRCPC or eligibility for exams by end of programme.

(List compiled June 1999 by Dr. David R. Bevan, Editor-in-Chief, Canadian Journal of Anesthesia, University of British Columbia, Vancotver, Britists Columbia) 